\title{
INJÚRIAS MECÂNICAS NA QUALIDADE PÓS-COLHEITA DE LIMA ÁCIDA 'TAHITI' ARMAZENADA SOB CONDIÇÃO AMBIENTE ${ }^{1}$
}

\author{
MARIA FERNANDA BERLINGIERI DURIGAN², BEN-HUR MATTIUZ², JOSÉ FERNANDO DURIGAN ${ }^{4}$
}

\begin{abstract}
RESUMO - O objetivo deste trabalho foi avaliar os efeitos de diferentes injúrias mecânicas na qualidade de limas ácidas 'Tahiti'. Foram testados três tipos de lesões: impacto, compressão e corte. Após esses tratamentos, os frutos foram armazenados em condições de ambiente ( $\left.25 \pm 1{ }^{\circ} \mathrm{C}, 65 \pm 5 \% \mathrm{UR}\right)$. As avaliações foram feitas a cada 3 dias, determinando-se atividade respiratória, aparência, perda de massa, $\mathrm{pH}$, teores de sólidos solúveis, acidez titulável e de ácido ascórbico. Durante o período de armazenamento, as lesões mostraram-se prejudiciais à qualidade, afetando distintamente os parâmetros químicos, a aparência e diminuindo os dias de possível comercialização dos frutos, principalmente naqueles submetidos ao corte e ao impacto. Estas injúrias também ocasionaram maior aumento na atividade respiratória dos frutos. A perda de massa fresca também foi observada durante este período e foi agravada quando os frutos foram submetidos ao corte. A injúria por impacto foi a mais prejudicial para a qualidade das limas ácidas 'Tahiti', comprometendo a aparência dos frutos aos 9 dias de armazenamento.
\end{abstract}

Termos para indexação: Citrus latifolia, compressão, impacto, corte, limão.

\section{MECHANICAL INJURIES ON POST HARVEST QUALITY OF 'TAHITI' LIME STORED UNDER ENVIROMENTAL CONDITIONS}

\begin{abstract}
This work aimed to evaluate the effects of different mechanical injuries on 'Tahiti' lime. Three types of injuries were tested: impact, compression and cut, then the fruits were stored under environmental conditions $\left(25^{\circ} \mathrm{C}, 65 \% \mathrm{RH}\right)$. The evaluations were done at each 3 days, when it was observed the respiratory activity and the appearance and it was determinated the loss of fresh mass and content of soluble solids, titratable acidity, ascorbic acid, and the $\mathrm{pH}$. During the storage, the injured fruit showed the worst quality, affecting the chemical parameters and the appearance and reduced the days of possible comercialization, mainly the ones submitted to cut and impact. Those injuries also caused a greater increase on the respiratory activity. Loss of weight was also observed during this period and it was increased when the fruits were submitted to cuts. The impact injury showed the worst results for the quality of 'Tahiti' limes, with undesirable appearance changes at the $9^{\text {th }}$ day of storage.
\end{abstract}

Index terms: Citrus latifolia, compression, impact, cut, lime.

\section{INTRODUÇÃO}

As perdas de produtos hortifrutigranjeiros, devido a danos físicos na cadeia entre o produtor e o consumidor, são estimadas em cerca de 30 a 40 \% (Barchi et al., 2002). Conforme Vigneault et al. (2002), do instante em que são colhidos até serem consumidos, os produtos hortícolas sofrem uma série de injúrias mecânicas que, dependendo da sensibilidade do produto, poderão causar danos que comprometerão a sua qualidade final, provocando perdas da ordem de 20 a $25 \%$ do total colhido.

Várias formas de contornar a falta de alimentos têm sido sugeridas, mas, na maioria dos casos, relega-se a planos secundários o combate a estas exorbitantes perdas. Prefere-se, em muitos casos, investir pesadamente na obtenção de cultivares, 10 - 15\% mais produtivas, a investir em novas embalagens ou tecnologias de armazenamento que permitam reduzir as perdas pós-colheita em 10 20\% (Mattiuz, 2002).

Aumentar a vida útil é o principal objetivo dos fisiologistas na pós-colheita, e o estudo dos problemas existentes compreende o conhecimento dos componentes que atuam no sistema, suas influências e as inter-relações entre eles (Prussia et al., 1986).

Dentre as causas de perdas pós-colheita, destacam-se aquelas devidas à ocorrência de injúrias mecânicas. Estas podem ser agrupadas em injúrias por impacto, compressão e corte. Tais injúrias ocasionam danos irreparáveis nos produtos, reduzindo sua vida útil e provocando uma conseqüente desvalorização comercial.

Estes danos mecânicos podem alterar as reações bioquímicas do produto, modificando-lhe a coloração, o sabor e a vida útil (Pantastico, 1979). Existem informações que tais injúrias ocasionam danos irreparáveis em frutas como goiabas (Mattiuz et al., 2002), cerejas (Burton \& SchultePason, 1987), melões 'Cantaloupe' (MacLeod et al., 1976) e maçãs (Parker et al., 1984), provocando aumentos na atividade respiratória e alterações químicas, com conseqüente redução na sua vida útil. O conhecimento dessas reações em frutas, como limões ou limas ácidas, assume uma grande importância, principalmente quando seu destino final é o consumo como fruta fresca ou minimamente processada.

A lima ácida 'Tahiti' é apreciada pelo consumidor na forma processada (sucos e caipirinha) por ser uma fruta rica em acidez, perfumada, de casca fina e sem sementes. Os produtores a valorizam por formar plantas vigorosas, com copa arredondada e, em especial, pela ausência de espinhos, facilitando assim a colheita (CEAGESP, 2000). O volume comercializado no CEAGESP vem crescendo e totalizou 66.017 toneladas em 2003. Naquele ano, foram exportadas 34.012 toneladas, o que representa US\$16.949.000 (AGRIANUAL, 2005).

O objetivo do presente trabalho foi avaliar os efeitos de diferentes injúrias mecânicas sobre a qualidade de limas ácidas 'Tahiti' .

\section{MATERIAL E MÉTODOS}

Utilizaram-se limas ácidas 'Tahiti’ procedentes do município de Taquaritinga-SP. A colheita destes frutos foi realizada de manhã e de forma inteiramente casualizada nas plantas do pomar. Os frutos foram colhidos no estádio de maturação considerado aceitável para exportação, sendo classificado como categoria 'Extra' pelo programa brasileiro para a melhoria dos padrões comerciais e embalagens de hortigranjeiros para limão (lima ácida) 'Tahiti', cujas características são: casca verde-escura e parcialmente lisa (coloração C2), e ausência de defeitos na casca, como ferimentos e manchas (CEAGESP, 2000).

Os frutos foram transportados cuidadosamente em caixas plásticas forradas com plástico tipo bolha e papel ao Laboratório de Tecnologia dos Produtos Agrícolas da FCAV/UNESP - Jaboticabal, distante $20 \mathrm{~km}$ do local da colheita. Os frutos foram novamente uniformizados quanto à coloração e deixados em repouso, por 1 hora, em local refrigerado $\left(20^{\circ} \mathrm{C}\right)$.

\footnotetext{
${ }^{1}$ (Trabalho 080/2005). Recebido: 06/05/2005. Aceito para publicação: 17/11/2005.

${ }^{2}$ Mestranda do Curso de Pós-Graduação em Produção Vegetal e bolsista Capes. mfbdurigan@yahoo.com.br.

${ }^{3}$ Prof. Dr. Departamento de Tecnologia. benhur@fcav.unesp.br.

${ }^{4}$ Prof. Titular do Departamento de Tecnologia. jfduri@fcav.unesp.br. FCAV - UNESP, Campus de Jaboticabal, Via de acesso Prof. Paulo Donato Castellane, s/n, 14884-900 Jaboticabal-SP.
} 
Foram avaliados três tipos de injúria mecânica: impacto, compressão e corte. Na injúria por Impacto, os frutos foram deixados cair de uma altura de 1,20 m sobre uma superfície maciça, plana e rígida. Cada fruto sofreu dois impactos, nos lados opostos de sua linha equatorial. Na Compressão, os frutos foram colocados sobre uma superfície plana e sobre esta um peso exerceu uma pressão de $49 \mathrm{~N}$, durante 20 minutos. Na injúria por Corte, os frutos foram submetidos a três incisões com $6 \mathrm{~cm}$ de comprimento por $2 \mathrm{~mm}$ de profundidade, feitos com lâminas, no sentido longitudinal e em ambos os lados do fruto.

As áreas lesionadas foram demarcadas, e os frutos, armazenados sob condições de ambiente $\left(25^{\circ} \mathrm{C}\right.$ e $65 \%$ UR).

$\mathrm{O}$ experimento foi conduzido em delineamento inteiramente casualizado, em esquema fatorial composto por três fatores: tipo de injúria (Controle, Impacto, Compressão e Corte), região com ou sem lesões e dias de armazenamento $(0 ; 3 ; 6 ; 9 ; 12 ; 15$ e 18 dias). Foram utilizadas três repetições para as determinações da evolução de $\mathrm{CO}_{2} \mathrm{e}$ para as análises químicas, e dez para a coloração.

As avaliações foram feitas a cada 3 dias, quando se determinou a aparência, mediante a atribuição de notas $(4=$ ótimo; $3=$ bom; $2=$ regular ou sem condições de comercialização; 1 = ruim ou impróprio para o consumo), as mudanças na massa fresca dos frutos, a coloração e análises químicas.

As avaliações de coloração foram efetuadas nas áreas injuriadas e não-injuriadas do mesmo fruto, utilizando-se do colorímetro Minolta CR 200b, para a obtenção dos valores de Luminosidade $\left(L^{*}\right)$, $a^{*} \mathrm{e} b^{*}$. Esses resultados permitiram calcular o ângulo de cor (Hue) e a Cromaticidade desta cor, conforme o recomendado por MINOLTA(1994). Para análises químicas, as amostras eram tomadas a cada três dias, comparando-se o suco dos frutos intactos com o suco dos frutos injuriados, nos quais foram separadas as áreas lesionadas das nãolesionadas. As amostras foram analisadas quanto aos seus conteúdos de ácido ascórbico (AA), acidez titulável (AT), sólidos solúveis (SS) (AOAC, 1997) e pH.

A produção de $\mathrm{CO}_{2}$ foi determinada colocando-se quatro frutos em recipiente com capacidade de 3 litros, hermeticamente fechado, por um período de 1 hora, sob as condições de ambiente. O conteúdo de $\mathrm{CO}_{2}$ da atmosfera do interior do recipiente era determinado, antes e imediatamente após este período, em cromatógrafo GC Finnigan 9001, e o resultado, expresso em mg de $\mathrm{CO}_{2} \cdot \mathrm{kg}^{-1} \cdot \mathrm{h}^{-1}$.

Os dados foram analisados pelo programa SAS (SAS INSTITUTE, 1999). A perda de massa fresca foi analisada comparandose a significância do paralelismo das retas obtidas, pelo Teste $\mathrm{T}$ (Neter et al., 1978).

\section{RESULTADOS E DISCUSSÃO}

A aparência externa dos frutos foi bastante prejudicada pela aplicação dos tratamentos injuriantes. Nos frutos do Controle, as limas ácidas mantiveram ótima aparência (nota 4) por até 15 dias de armazenamento, enquanto as submetidas às injúrias mecânicas se encontravam bastante prejudicadas no $6^{\circ}$ dia. Os frutos submetidos ao Corte apresentaram manchas escuras, características de oleocelose, algumas horas após o lesionamento. Frutos submetidos ao Impacto mostraram-se sem condições de comercialização após 9 dias de armazenamento. Nos submetidos à Compressão, esta condição foi atingida entre o $12^{\circ}$ e o $15^{\circ}$ dia de armazenamento quando $70 \%$ dos frutos apresentaram notas abaixo de 3 . Internamente, os frutos intactos permaneceram ótimos até o final do experimento, enquanto os frutos injuriados se apresentavam sem condições de comercialização a partir do $9^{\circ}$ dia de armazenamento. A partir deste dia, estas limas receberam nota 2 (ruim) para os tratamentos Corte e Compressão, e nota 1 (péssimo) para os frutos do Impacto, que apresentaram sintomas de podridão estilar devido ao rompimento das vesículas de suco e conseqüente encharcamento das células que compõem as extremidades dos limões.

Os frutos do Controle apresentaram atividade respiratória média de $19,33 \mathrm{mg}$ de $\mathrm{CO}_{2} \cdot \mathrm{kg}^{-1} \cdot \mathrm{h}^{-1}$. As injúrias mecânicas aumentaram a atividade respiratória (Figura 1). Para a Compressão, este aumento foi de 1,5; para o Corte, apesar das grandes variações, este incremento respiratório foi 2,2 vezes; e para o Impacto esta atividade triplicou.

Esses resultados reafirmam aqueles encontrados por Burton \& Schulte-Pason (1987), que evidenciaram aumento na evolução de $\mathrm{CO}_{2}$ de cerejas submetidas a várias intensidades de impacto. Estes autores atribuíram esse incremento respiratório à descarboxilação do ácido málico, que teria extravasado das células danificadas no local de ocorrência das injúrias. Na injúria por impacto, os frutos apresentaram maior produção de $\mathrm{CO}_{2}$ que nas demais injúrias. Isto evidencia que esta lesão é grave, provavelmente devido a um maior rompimento de células

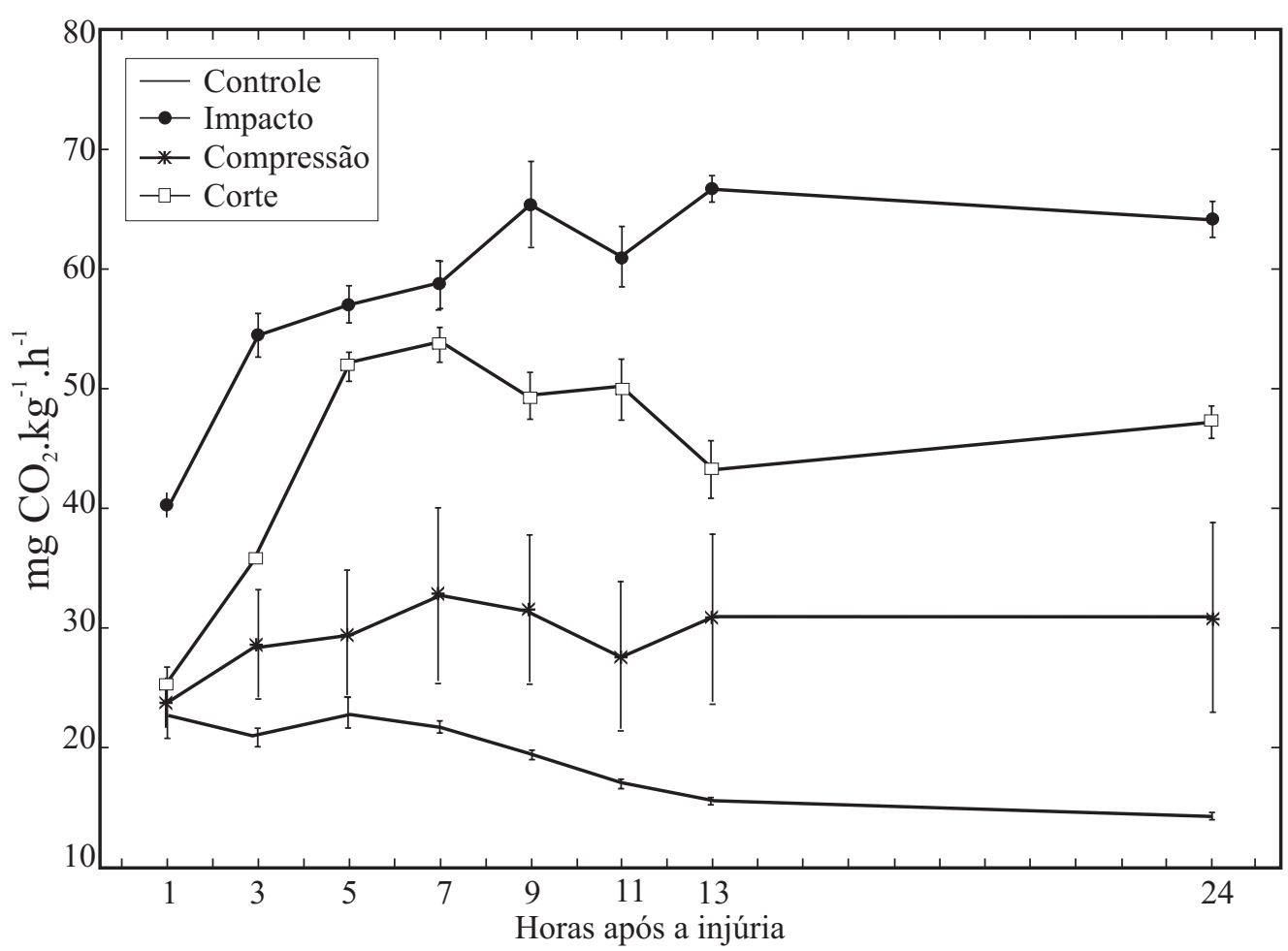

FIGURA 1 - Evolução da atividade respiratória de limas ácidas ‘Tahiti’ submetidas a diferentes injúrias mecânicas e armazenadas a $25^{\circ} \mathrm{C}$ e $65 \%$ UR. Barras verticais representam o desvio-padrão da média $(\mathrm{n}=3)$. 
por ocasião da injúria, o que teria disponibilizado os ácidos presentes no vacúolo para o sistema respiratório, na tentativa de reparar o dano causado (Mattiuz \& Durigan, 2001a). O impacto também foi responsável por aumentos na atividade respiratória de melões Cantaloupe (MacLeod et al., 1976), de maçãs (Parker et al., 1984), de tomates (Moretti et al., 1999) e de goiabas (Mattiuz, 2002).

As injúrias levaram os frutos a perderem massa fresca com maior intensidade que os frutos do Controle, durante o período de armazenamento (Tabela 1). Dentre as injúrias, a que levou à maior perda de massa foi o Corte, seguida do Impacto e da Compressão. Este tratamento também foi o mais prejudicial para mangas 'Palmer', enquanto, para mangas 'Keitt' (Durigan, 2003), pêssego (Kasat, 2003) e abacate (Sanches et al., 2004), a maior perda de massa ocorreu no Impacto.

TABELA 1 - Equações representativas da evolução da perda de massa fresca por limas ácidas 'Tahiti', submetidas a diferentes injúrias mecânicas e armazenadas por 18 dias a $25^{\circ} \mathrm{C}$ e $65 \%$ UR, e comparação entre as equações através do teste $\mathrm{T}$, quanto ao paralelismo.

\begin{tabular}{lccc}
\hline Tratamentos & $\mathrm{y}=\mathrm{a}+\mathrm{bx}$ & $\mathrm{R}$ & Paralelismo \\
\hline Controle & $\mathrm{y}=84,6454-0,8406 \mathrm{x}$ & $-0,9985^{* *}$ & $\mathrm{~d}$ \\
Impacto & $\mathrm{y}=89,3593-0,9336 \mathrm{x}$ & $-0,9946^{* *}$ & $\mathrm{~b}$ \\
Compressão & $\mathrm{y}=91,8936-0,8517 \mathrm{x}$ & $-0,9889^{* *}$ & $\mathrm{c}$ \\
Corte & $\mathrm{y}=84,4657-1,1538 \mathrm{x}$ & $-0,9950^{* *}$ & $\mathrm{a}$ \\
\hline $\mathrm{y}=$ perda de massa fresca $(\mathrm{g}) ; \mathrm{x}=$ tempo de armazenamento (dia).
\end{tabular}

A análise estatística referente aos parâmetros químicos demonstraram significância somente para o efeito principal "tipo de injúria", sendo suas médias apresentadas na Tabela 2. Houve diferenças significativas entre os valores médios dos parâmetros avaliados, com exceção da relação SS/AT, que se manteve constante (Tabela 2). O Impacto reduziu significativamente os teores de SS, AT e AA, refletindo seu maior efeito estressante a esta fruta, ou seja, promoveu uma senescência mais rápida, quando comparado ao Corte e à Compressão, que também causaram efeitos senescentes, porém menos evidentes. Mattiuz \& Durigan (2001a) observaram o mesmo para goiabas assim como Sanches et al. (2004) para abacates e Moretti et al. (1999) para tomates. Neste último, a redução dos teores foi atribuída à diminuição na concentração de ácidos orgânicos. O pH refletiu a acidez titulável, e os teores de ácido ascórbico só foram afetados significativamente pelo Impacto, reafirmando o efeito negativo do mesmo. O menor valor para o ácido ascórbico evidencia a perda do poder antioxidante e protetor deste ácido, mostrando que os frutos e as regiões injuriadas já estavam em estado de deterioração mais avançado que nos outros tratamentos. Moretti et al. (1999) também observaram grande diminuição nos teores de vitamina $\mathrm{C}$ em tecidos injuriados de tomates. $\mathrm{O}$ primeiro autor constatou que o tecido locular não injuriado de tomates apresentou $17 \%$ mais AA que o tecido injuriado. Seus dados permitiram sugerir que os sistemas protetores antioxidantes associados com o ácido ascórbico são danificados pelas injúrias mecânicas, permitindo a degradação oxidativa irreversível do ácido ascórbico a ácido 2,3 diceto L-gulônico (Burton \& Schulte-Pason, 1987).

Houve uma evolução significativa para as variáveis

TABELA 2 - Qualidade avaliada por diferentes parâmetros químicos, de limas ácidas 'Tahiti' submetidas a diferentes injúrias mecânicas e armazenadas por 18 dias a $25^{\circ} \mathrm{C}$ e $65 \%$ UR.

\begin{tabular}{lccccc}
\hline Tratamento & SS & AT & SST/AT & pH & AA \\
\hline Controle & $8,4 \mathrm{a}$ & $7,9 \mathrm{a}$ & $1,07 \mathrm{a}$ & $2,17 \mathrm{bc}$ & $54,60 \mathrm{a}$ \\
Impacto & $7,6 \mathrm{c}$ & $7,0 \mathrm{c}$ & $1,10 \mathrm{a}$ & $2,22 \mathrm{a}$ & $51,36 \mathrm{~b}$ \\
Compressão & $7,9 \mathrm{bc}$ & $7,4 \mathrm{~b}$ & $1,08 \mathrm{a}$ & $2,16 \mathrm{c}$ & $56,83 \mathrm{a}$ \\
Corte & $8,1 \mathrm{ab}$ & $7,4 \mathrm{~b}$ & $1,11 \mathrm{a}$ & $2,19 \mathrm{ab}$ & $56,59 \mathrm{a}$ \\
\hline
\end{tabular}

SS = sólidos solúveis $\left({ }^{\circ} \mathrm{Brix}\right) ; \mathrm{AT}=$ acidez titulável (g.ac.cítrico. $\left.100 \mathrm{~mL}^{-1}\right) ; \mathrm{AA}$ = ácido ascórbico $\left(\mathrm{mg} \cdot 100 \mathrm{~mL}^{-1}\right)$. Para cada variável, médias seguidas de mesma letra não diferem significativamente entre si, pelo teste de Tukey $(\mathrm{P}<0,05)$ dependentes Luminosidade, Ângulo de Cor (Hue) e Cromaticidade, ao longo do tempo de armazenamento (Tabela 3 ), associada à evolução da cor dos frutos. A injúria por Impacto levou os limões a uma maior perda na cor verde, principalmente nas regiões lesionadas dos frutos. Kasat (2003) também observou esta diferença entre as regiões lesionadas e não-lesionadas de pêssegos 'Aurora-1' quando submetidos a diferentes injúrias mecânicas. $\mathrm{O}$ aumento da luminosidade, associado ao aumento na cromaticidade e à diminuição do ângulo de cor, revela que houve perda da cor verde, fazendo com que os frutos adquirissem tonalidade amarelada, resultante da redução nos conteúdos de clorofila (Mattiuz $\&$ Durigan, 2001b). Este fato deve-se, provavelmente, à degradação da clorofila pela enzima clorofilase, por modificação no $\mathrm{pH}$ ou por sistemas oxidativos da magnitude da injúria aplicada.

TABELA 3 - Luminosidade, Ângulo de cor e Cromaticidade da parte externa de limas ácidas 'Tahiti', submetidas a diferentes injúrias mecânicas e armazenadas a $25^{\circ} \mathrm{C}$ e $65 \%$ UR, por 18 dias.

\begin{tabular}{lccc}
\hline \multicolumn{1}{c}{ Variável } & Luminosidade & $\begin{array}{c}\text { Ângulo de } \\
\text { Cor }\end{array}$ & Cromaticidade \\
\hline $\begin{array}{l}\text { Tipo de injúria } \\
\text { Controle }\end{array}$ & $54,080 \mathrm{~b}$ & $127,256 \mathrm{a}$ & $41,776 \mathrm{a}$ \\
Impacto & $57,842 \mathrm{a}$ & $121,803 \mathrm{~b}$ & $44,633 \mathrm{a}$ \\
Compressão & $52,094 \mathrm{~b}$ & $125,932 \mathrm{a}$ & $40,247 \mathrm{~b}$ \\
Corte & $51,773 \mathrm{~b}$ & $125,628 \mathrm{a}$ & $33,060 \mathrm{c}$ \\
\hline DMS & 3,558 & 2,682 & 3,649 \\
\hline Fruto & & & \\
Área não lesionada & $53,307 \mathrm{a}$ & $127,114 \mathrm{a}$ & $40,710 \mathrm{a}$ \\
$\quad$ Área lesionada & $54,601 \mathrm{a}$ & $123,267 \mathrm{~b}$ & $39,417 \mathrm{a}$ \\
\hline DMS & 1,913 & 1,442 & 1,962 \\
\hline Dias após a injúria & & & \\
0 & $50,360 \mathrm{~d}$ & $135,708 \mathrm{a}$ & $34,233 \mathrm{~d}$ \\
3 & $50,648 \mathrm{~cd}$ & $126,259 \mathrm{~b}$ & $38,569 \mathrm{bcd}$ \\
6 & $51,918 \mathrm{bcd}$ & $124,886 \mathrm{bc}$ & $36,044 \mathrm{~cd}$ \\
9 & $53,383 \mathrm{BCD}$ & $124,139 \mathrm{bc}$ & $41,079 \mathrm{abc}$ \\
12 & $56,040 \mathrm{abc}$ & $121,302 \mathrm{~cd}$ & $42,865 \mathrm{ab}$ \\
15 & $56,765 \mathrm{ab}$ & $123,464 \mathrm{~cd}$ & $43,564 \mathrm{ab}$ \\
18 & $59,876 \mathrm{a}$ & $119,460 \mathrm{~d}$ & $45,420 \mathrm{a}$ \\
\hline DMS & 5,419 & 4,084 & 5,557 \\
\hline
\end{tabular}

$\overline{\text { Para cada variável, médias seguidas de mesma letra não diferem significativamente }}$ entre si, pelo teste de Tukey $(\mathrm{P}<0,05)$

\section{CONCLUSÕES}

Houve perda significativa da qualidade das limas ácidas 'Tahiti' em função das diferentes injúrias mecânicas, principalmente naquelas submetidas às injúrias por impacto.

\section{AGRADECIMENTOS}

À FAPESP (Proc. no 03/08592-2) e à CAPES, pelo auxílio financeiro.

\section{REFERÊNCIAS}

AGRIANUAL 2005: anuário da agricultura brasileira. São Paulo: FNP Consultoria e Comércio, 2005. p.306, 306-311.

AOAC. Official methods of analysis of the Association of Official Analytical Chemists International. $16^{\text {th }}$ ed. Washington: Patricia Cummiff, 1997. v.2, cap.37. (Métodos 967.21, 943.03, 932.12).

BARCHI, G. L.; BERARDINELLI, A.; GUARNIERI, A.; RAGNI, L.; TOTARO FILA, C. Damage to loquats by vibration-simulating intrastate transport. Biosystems Engineering, London, v. 82, n. 3, p. 305-312, 2002.

BURTON, C.L.; SCHULTE-PASON, N.L. Carbon dioxide as an indicator of fruit impact damage. HortScience, Alexandria, v. 22, n. 2, p. 281- 
$2,1987$.

CEAGESP. Centro de Qualidade em Horticultura. Classificação do limão (Lima ácida) Tahiti (Citrus latifolia Tanaka). São Paulo, 2000. Fôlder. (Programa Brasileiro para a Melhoria dos Padrões Comerciais e Embalagens de Hortigranjeiros).

DURIGAN, M.F.B. Injúrias mecânicas na qualidade de mangas 'Palmer' e 'Keitt'. 2003. 63f. Dissertação (Graduação em Agronomia) Faculdade de Ciências Agrárias e Veterinarias - Universidade Estadual Paulista, Jaboticabal, 2003.

KASAT, G.F. Efeito de injúrias mecânicas na qualidade pós-colheita de pêssegos 'Aurora-1'. 2003. 47f. Dissertação (Graduação em Agronomia) - Faculdade de Ciências Agrárias e Veterinárias, Universidade Estadual Paulista, Jaboticabal, 2003.

KAYS, J.S. Postharvest physiology of perishable plant products. New York: Van Nostrand Reinhold, 1991. 453p.

MacLEOD, R.F.; KADER, A.A.; MORRIS, L.L. Damage to fresh tomatoes can be reduced. California Agriculture, Berkeley, v. 30, n. 12, p. 10-12, 1976.

MATTIUZ, B.H.; BISCEGLI, C.A.; DURIGAN, J.F. Aplicações da tomografia de ressonância magnética nuclear como método nãodestrutivo para avaliar os efeitos de injúrias mecânicas em goiabas 'Paluma' e 'Pedro Sato'. Revista Brasileira de Fruticultura, Jaboticabal, v.24, n.3, p.641-643, 2002.

MATTIUZ, B.H.; DURIGAN, J.F. Efeito de injúrias mecânicas no processo respiratório e nos parâmetros químicos de goiabas 'Paluma' e 'Pedro Sato'. Revista Brasileira de Fruticultura, Jaboticabal, v.23, n.2, p.282-287, 2001a.

MATTIUZ, B.H.; DURIGAN, J.F. Efeito de injúrias mecânicas na firmeza e coloração de goiabas das cultivares 'Paluma' e 'Pedro Sato'. Revista Brasileira de Fruticultura, Jaboticabal, v.23, n.2, p.277281, 2001b.

MATTIUZ, B.H. Injúrias mecânicas e processamento mínimo de goiabas: fisiologia e qualidade pós-colheita. 2002. 120f. Tese
(Doutoramento em Produção Vegetal) - Faculdade de Ciências Agrárias e Veterinárias, Universidade Estadual Paulista, Jaboticabal, 2002.

MINOLTA. Precise color communication: color control from feeling to instrumentation. Japão, 1994. 49p.

MORETTI, C.L.; SARGENT, S.; HUBER, D.J. Delayed ripening does not alleviate symptoms of internal bruising in tomato fruit. Proceedings of the Florida State Horticultural Society, Winter Haven, v.112, p.169-171. 1999

NETER, J.; WASSERMAN, W.; WHIMORE, G.A. Applied linear statistical models. Massachussets: Allyn and Bacon, 1978. 745p.

PANTASTICO, ER.B. Fisiología de la postrecoleccion, manejo y utilización de frutas y hortalizas tropicales y subtropicales. Mexico: Compañia Editorial Continental, 1979. 663 p.

PARKER, M.L.; WARDOWSKI, W.F.; DEWEY, D.H. A damage test for oranges in a commercial packing house line. Proceedings of the Florida State Horticultural Society, Winter Haven, v.97, p.136-137. 1984

PRUSSIA, S.E.; JORDAN, J.L.; SHEWFELT, R.L.; BEVERLY, R.B. A systems approach for interdisciplinary postharvest research on horticulture crops. Georgia: Experimental State Research Report, Athens, 1986.514p.

SANCHES, J.; BISCEGLI, C.I.; DURIGAN, J.F.; DURIGAN, M.F.B.; SIMÕES, M.L.; LOPES, W.T.L. Efeitos de injúrias mecânicas em abacates 'Hass' e 'Quintal'. In: CONGRESSO BRASILEIRO DE FRUTICULTURA, 18., 2004, Florianópolis. Anais... CD-ROM.

SAS INSTITUTE. SAS System for Microsoft windows: release 8.02. Cary, NC, USA, 1999.

VIGNEAULT, C.; BORDINT, M. R.; ABRAHÃO, R. F. Embalagem para frutas e hortaliças. In: CORTEZ, L. A. B.; HONÓRIO, S. L.; MORETTI, C. L. Resfriamento de frutas e hortaliças. Brasília: Embrapa Informação Tecnológica, 2002.p. 95-121. 